\title{
Colon-specific pulsatile drug release provided by electrospun shellac nanocoating on hydrophilic amorphous composites
}

This article was published in the following Dove Press journal: International Journal of Nanomedicine

\author{
Yao-Yao Yang ${ }^{1, *}$ \\ Zhe-Peng Liu'* ${ }^{2} *$ \\ Deng-Guang $\mathrm{Yu}^{\prime}$ \\ Ke Wang' \\ Ping Liu' \\ Xiaohong Chen'
}

'School of Materials Science and Engineering, University of Shanghai for Science and Technology, Shanghai, China; ${ }^{2}$ School of Medical Instrument and Food Engineering, University of Shanghai for Science and Technology, Shanghai, China

*These authors contributed equally to this work
Correspondence: Deng-Guang Yu; Xiaohong Chen

School of Materials Science and Engineering, University of Shanghai for Science and Technology, No 516, Jun-Gong Road, Shanghai 200093, China $\mathrm{Tel} / \mathrm{fax}+862155270632$ Email ydg017@usst.edu.cn; cxh992@163.com
Background: Colon-specific pulsatile drug release, as a combined drug controlled-release model, is a useful drug delivery manner for a series of diseases. New nanomedicines and related preparation methods are highly desired.

Methods: With diclofenac sodium (DS) as a model drug, a new type of structural nanocomposite (SC), in which composite polyvinylpyrrolidone (PVP)-DS core was coated by shellac, was fabricated via modified coaxial electrospinning. For comparison, traditional PVP-DS monolithic hydrophilic nanocomposites (HCs) were generated using a traditional blending process. Scanning electron microscopy (SEM), transmission electron microscopy (TEM), X-ray diffraction (XRD), attenuated total reflectance-Fourier transform infrared (ATR-FTIR), water contact angle (WCA), and in vitro dissolution and ex vivo permeation tests were conducted to characterize the composites.

Results: SEM images demonstrated that both composites were linear nanofibers with smooth surface morphology and cross sections. TEM disclosed that the SCs had a thin shellac sheath layer of approximately $12 \mathrm{~nm}$. XRD and ATR-FTIR results demonstrated that the crystalline DS was converted into amorphous composites with PVP because of favorable secondary interactions. WCA and in vitro dissolution tests demonstrated that the sheath shellac layers in SC could resist acid conditions and provide typical colon-specific pulsatile release, rather than a pulsatile release of $\mathrm{HC}$ under acid conditions. Ex vivo permeation results demonstrated that the SCs were able to furnish a tenfold drug permeation rate than the DS particles on the colon membrane.

Conclusion: A new SC with a shellac coating on hydrophilic amorphous nanocomposites could furnish a colon-specific pulsatile drug release profile. The modified coaxial process can be exploited as a useful tool to create nanocoatings.

Keywords: modified coaxial electrospinning, nanocoating, colon-targeted, pulsatile release, structural composites

\section{Introduction}

After half a century of pharmaceutical development, research on drug delivery is gradually extricating itself from the traditional models of drug controlled release, in which drug-release profiles often rely solely on the chemical and physical properties of pharmaceutical excipients. ${ }^{1-3}$ New combined models are frequently developed, in which complicated drug controlled-release profiles (such as multiple-phase release, dual release, controlled release of multiple active ingredients in one dosage form, simultaneous control release of time and initial place, or specific release of place and release rate) are provided for improved therapeutic effects. ${ }^{4-6}$ Among them, colon-specific (or colon-targeted) pulsatile drug delivery, which allows a time- and site-specific 
release of drugs in the colon after a predetermined lag time, is a new advanced drug controlled-release model. It is useful in the treatment of common diseases, such as angina pectoris, bronchial asthma, allergic rhinitis, hypertension, rheumatoid arthritis, and colorectal cancer. ${ }^{7}$

Many efforts have been dedicated to drug colon-targeted pulsatile release through traditional methods on solid dosage forms. ${ }^{8-11}$ Vemula and Katkum reported coated tablets of ketorolac tromethamine, which were prepared using double-compression coating method. ${ }^{8}$ Similarly, Tung et al investigated colon-specific targeted tablets of metronidazole through a pectin/hydroxypropyl methylcellulose dry powder coating. ${ }^{9}$ Vemula also reported double-compression-coated mini-tablets for pulsatile colonic release of flurbiprofen, ${ }^{10}$ as well as colon-specific pH- and time-dependent tablets. ${ }^{11}$ However, only a few studies have reported about colonspecific pulsatile nanodrug delivery systems using advanced technologies.

Nanomedicine has increasingly relied on complex nanostructures rather than nanosized products to achieve super functional performances. ${ }^{12-14}$ Among all kinds of nanostructures and nanodevices, core-shell (or core-sheath) and Janus are the most fundamental structural characteristics, which are frequently explored to develop novel functional nanomaterials. ${ }^{15-19}$ In particular, core-shell nanostructures (with extended nanocoating and hollow nanotube) have found a major application in a wide variety of fields. ${ }^{20}$ Numerous publications have demonstrated that they are popular for tailoring compositions and space distributions within nanoproducts. ${ }^{21,22}$ Core-shell nanostructures should have great potential to develop materials for colon-specific (or colon-targeted) pulsatile drug-release profiles.

Core-shell nanostructures can be generated using topdown and bottom-up methods. ${ }^{23,24}$ Electrospinning, as a typical top-down technique, is presently booming because of its ability to generate all types of complex nanostructures with the macrostructural spinneret as a template. ${ }^{25,26}$ For example, core-sheath nanofibers can be prepared using concentric spinneret, trilayer nanofibers can be obtained using triaxial spinneret, and Janus nanofibers can be created using side-by-side spinneret. ${ }^{27-29}$

In this study, a colon-specific pulsatile structural nanocomposite (SC) was fabricated using modified coaxial electrospinning. In the new SC, a thin shellac layer was coated on an amorphous hydrophilic nanocomposite (HC) consisting of polyvinylpyrrolidone (PVP) and drug, with the shellac layer ensuring a colon-targeted effect and the coated HC providing the pulsatile release of a poorly water-soluble drug, namely, diclofenac sodium (DS). Monolithic PVP-DS nanocomposites were fabricated through a blending process for comparison. DS, a typical nonsteroidal anti-inflammatory drug, has potent antipyretic, analgesic, and anti-inflammatory properties. However, it may result in severe side effects with high toxicity. Oral colon-specific pulsatile release is not only expected to provide an enhanced therapeutic effect but also minimizes possible side effects in the stomach. ${ }^{30}$

Macromolecules from nature including protein, polysaccharide, and resin are popular as medical matrices, additives, and adhesive glue. ${ }^{31,32}$ For drug delivery applications, a wide variety of cellulose and their derivatives have been exploited as drug carriers because of their fine compatibility, safety, biodegradability, and amorphousness. Other types of new natural macromolecules are continuously discovered for potential applications in this region, particularly those with unique solubility and which can be exploited for manipulating specific drug delivery characteristics such as zein and shellac. Shellac is insoluble under acid conditions but soluble in a neutral environment. Thus, it can be coated on HCs so that SCs can resist acid erosion.

\section{Materials and methods Materials}

DS and shellac were obtained from Hubei Biocause Pharm. Co., Ltd. (Wuhan, China) and Shanghai Wan-Jiang BioTechnol. Co., Ltd. (Shanghai, China), respectively. PVP $\left(\mathrm{M}_{\mathrm{w}}=58,000\right.$, PVP K30) was purchased from Sigma-Aldrich (Shanghai, China). Anhydrous ethanol was obtained from Shanghai Chem. Reag. Co., Ltd. (Shanghai, China). All other chemicals were of analytical grade. Water was double distilled before use.

\section{Fabrication}

All processes were implemented through an electrospinning apparatus, which consisted of two syringe pumps (KDS100, Cole-Parmer, USA), a high-voltage generator (ZGF60 kV/2 mA, Wuhan Hua-Tian Co., China), a homemade coaxial spinneret, and a fiber collector.

After some preliminary experiments, two composite nanofibers were prepared. One was nanofiber F1, which was an $\mathrm{HC}$ fabricated by a single-fluid blending process. A blending solution composed of 35\% PVP (weight/volume [w/v]) and $5 \% \mathrm{DS}(\mathrm{w} / \mathrm{v})$ in ethanol was treated under an applied voltage of $12 \mathrm{kV}$, fiber-collected distance of $15 \mathrm{~cm}$, and a flow rate of $1.0 \mathrm{~mL} / \mathrm{h}$.

The other was nanofiber F2, which was an SC fabricated through modified coaxial electrospinning. A blending 
solution comprising 35\% PVP (w/v) and 5\% DS (w/v) in ethanol was utilized as the core fluid. The sheath fluid was an unspinnable dilute shellac solution with a concentration of $10 \%(\mathrm{w} / \mathrm{v})$. The experimental conditions were as follows: applied voltage of $12 \mathrm{kV}$, fiber-collected distance of $15 \mathrm{~cm}$, core flow rate of $0.8 \mathrm{~mL} / \mathrm{h}$, and sheath ethanol flow rate of $0.2 \mathrm{~mL} / \mathrm{h}$.

\section{Morphology and nanostructure}

The prepared nanofibers were evaluated using a scanning electron microscope (SEM; FEI Quanta450FEG, USA). Prior to microscopy, the fiber mats were sputter coated with platinum for $90 \mathrm{~s}$. The cross-section samples were prepared by manually breaking fiber mats, which were inserted into liquid nitrogen for over $15 \mathrm{~min}$. The inner structure of nanofibers was investigated using an $\mathrm{H}-800$ transmission electron microscope (TEM; Hitachi, Japan).

\section{Physical forms and compatibility}

$\mathrm{X}$-ray diffraction (XRD) experiments were conducted using a Bruker X-ray powder diffractometer (Bruker-AXS, Karlsruhe, Germany). The raw drug, polymer powders, and nanofiber samples were detected at a $2 \theta$ angle range of $5^{\circ}-60^{\circ}$.

The raw PVP/DS/shellac powders and their composites were subjected to attenuated total reflectance-Fourier transform infrared (ATR-FTIR) analyses through a spectrometer (Spectrum 100, PerkinElmer, Billerica, MA, USA).

\section{Hydrophilic property and drug-loading capability}

The hydrophilicity of the HCs and SCs was evaluated using a drop shape analysis instrument (DSA100, Krüss GmbH, Hamburg, Germany) by measuring their surface water contact angle (WCA). About $3 \mu \mathrm{L}$ of $\mathrm{pH} 2.0 \mathrm{HCl}$ solution or $\mathrm{pH} 7.0$ PBS $(0.1 \mathrm{~mol} / \mathrm{L})$ was deposited onto the surface of a sample, and the receded processes of the droplet were recorded.

\section{Encapsulation efficiency of DS in the nanofibers}

To free the contained DS molecules from the SCs and HCs, $0.3 \mathrm{~g}$ was dissolved in $100 \mathrm{~mL}$ of ethanol. Approximately $0.2 \mathrm{~mL}$ of the resultant solution was diluted to $200 \mathrm{~mL}$ using PBS and then filtered through a $0.22-\mu \mathrm{m}$ membrane (Millipore, MA, USA) for UV-vis detection at $276 \mathrm{~nm}$ with a spectrophotometer (UV-2102PC, Unico Instrument Co., Ltd., Shanghai, China) in accordance with the reported protocols in the Chinese Pharmacopoeia. Encapsulation efficiency (E) was calculated using the following equation:

$$
\mathrm{E}=\frac{C_{d}}{C_{c}} \times 100 \%
$$

where $C_{d}$ and $C_{c}$ are the experimentally detected and theoretically calculated drug concentrations, respectively.

\section{In vitro dissolution tests}

An RCZ-8A dissolution apparatus (Tian-Jin University Radio Factory, Tian-Jin, China) was exploited to carry out the in vitro dissolution tests. Samples containing an equivalent of $40 \mathrm{mg}$ of DS (ie, $0.30 \mathrm{~g}$ of F1 and $0.34 \mathrm{~g}$ of F2) were placed in dissolution cells containing dissolution medium $(900 \mathrm{~mL}$ of $\mathrm{HCl}$ for the first $2 \mathrm{~h}$ and $900 \mathrm{~mL}$ of PBS for the later time period), which were maintained at a constant temperature of $37^{\circ} \mathrm{C} \pm 1^{\circ} \mathrm{C}$ and a rotation rate of $50 \mathrm{rpm}$. Approximately $5.0 \mathrm{~mL}$ aliquots were withdrawn from the dissolution media to detect drug concentrations at predetermined time points. The withdrawn volume was replaced with $5.0 \mathrm{~mL}$ of fresh medium to maintain the bulk dissolution media at a constant volume. All experiments were repeated 6 times.

\section{Ex vivo permeation tests}

An RYJ-6A diffusion test apparatus (Shanghai Huang-Hai Drug Control Instrument Co., Ltd., Shanghai, China) was exploited to conduct the ex vivo permeation experiments. The diffusion area of each cell was $2.60 \mathrm{~cm}^{2}$. Each donor and receptor compartment was filled with 1 and $7.2 \mathrm{~mL}$ of PBS, respectively. A Teflon-coated magnetic bead in the receptor compartment was rotated at $50 \mathrm{rpm}$. The large pig intestines (obtained from Bao-Shan Jiang-Wan slaughterhouse, Shanghai, China) were cleaned using physiologic saline $(\mathrm{NaCl}$ $0.9 \%, \mathrm{w} / \mathrm{v}$ ) before the colonic membranes were peeled off. The diffusion cells were covered with the peeled membranes and and equilibrated for $30 \mathrm{~min}$ at $37^{\circ} \mathrm{C}$ before permeation experiments. The SC $(85 \mathrm{mg})$ was placed on the mucosal surfaces. At a predetermined time point, a $1 \mathrm{~mL}$ aliquot was withdrawn from the receptor compartment, and $1 \mathrm{~mL}$ of fresh PBS was added. The aliquots were filtered through a $0.22-\mu \mathrm{m}$ film (Millipore, Billerica, MA, USA) before UV detection. All measurements were conducted in triplicate. Permeation experiments were performed with $10 \mathrm{mg}$ of raw DS as a control.

\section{Results and discussion Modified coaxial electrospinning}

In 2002, Locertales et al first reported coaxial electrohydrodynamic atomization. ${ }^{33}$ In 2004, coaxial electrospinning was 
thought to be one of the most important breakthroughs in this field. ${ }^{34}$ In 2008, Morghe and Gupta declared that sheath fluids should be spinnable and have sufficient viscosity to eliminate the interfacial tension between the two working fluids for a successful coaxial process. ${ }^{35}$ However, Yu et al corrected this concept to develop a series of modified coaxial and triaxial electrospinning processes, which are characterized by the unspinnable sheath working fluid. ${ }^{36-40}$ A schematic of the modified coaxial system is shown in Figure 1A, which consists of a high-voltage generator, two pumps, a fiber collector, and a spinneret. When the shell fluid $\left(F_{\mathrm{s}}=0\right)$ is switched off, traditional single-fluid blending electrospinning can be implemented using the same system.

Shellac, as a natural polymer, has been demonstrated to be electrospinnable. However, a high concentration of up to $75 \%(\mathrm{w} / \mathrm{v})$ is needed to ensure a bead-free linear structure. ${ }^{41}$ Thus, a thick sheath layer was inevitable when traditional coaxial electrospinning was exploited to prepare the coresheath nanostructures, where an electrospinnable sheath solution was applied. Thus, modified coaxial electrospinning can exert its advantages over traditional coaxial processes in that a uniform thin layer can be generated on the core composites. The thin layer is useful for the pulsatile release profile because of the rapid removal of the shellac layer when the nanofibers were transferred into a neutral condition. SCs with thin shellac nanocoating were designed using the modified coaxial process (Figure 1B). The thin shellac layer was expected to resist the acid conditions in the stomach, and the inner PVP-based composites could be dissolved all at once when they encountered water. The sheath and core parts acted synergistically to provide colon-specific pulsatile release.
Figure 2 shows the electrospinning systems (Figure 2A), arrangement of the apparatuses (Figure 2B and $\mathrm{C}$ ), and working processes of single-fluid blending electrospinning (Figure 2D1 and D2) and modified coaxial electrospinning (Figure 2E1 and E2). An image of the homemade coaxial spinneret is shown in the inset of Figure 2A. The syringe contained the core fluid connected with the spinneret directly, whereas the sheath fluid was pumped to the spinneret through a silicon tube (Figure 2B). An alligator clip was exploited to transfer the electrostatic energy to the working fluids (Figure 2C).

Under the selected conditions and no sheath fluid, a typical single-fluid blending electrospinning process is shown in Figure 2D1, where a straight fluid jet followed by numerous bending and whipping circles was obvious. An enlarged Taylor cone is shown in Figure 2D2. For the sheath shellac solution provided to conduct modified coaxial electrospinning, the working processes and compound Taylor cone are illustrated in Figure 2E1 and E2, respectively. The shellac solution had a reddish brown color. Under strong camera flash, the sheath solution exhibited a hazel color, with a discerned colorless core solution at the tip of the Taylor cone (Figure 2E2). Both single-fluid electrospinning for preparing $\mathrm{HC}$ and the modified coaxial process for preparing $\mathrm{SC}$ were able to run smoothly and continuously, generating nanofibers of high quality.

\section{Morphology and nanocoating}

The SEM images of the surface morphologies of HCs and SCs are shown in Figure $3 \mathrm{~A}$ and B, respectively. Both HCs and SCs displayed linear morphology without any beads or spindles. The monolithic HC F1 nanofibers had an average
A

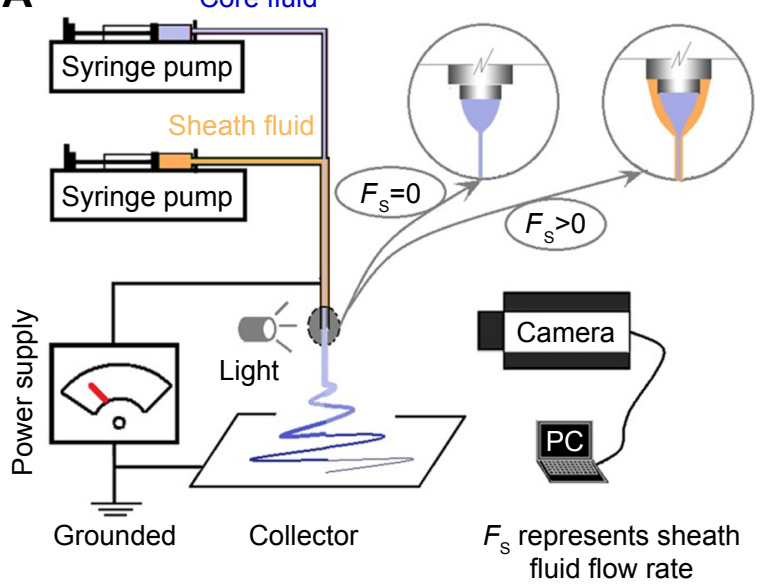

B

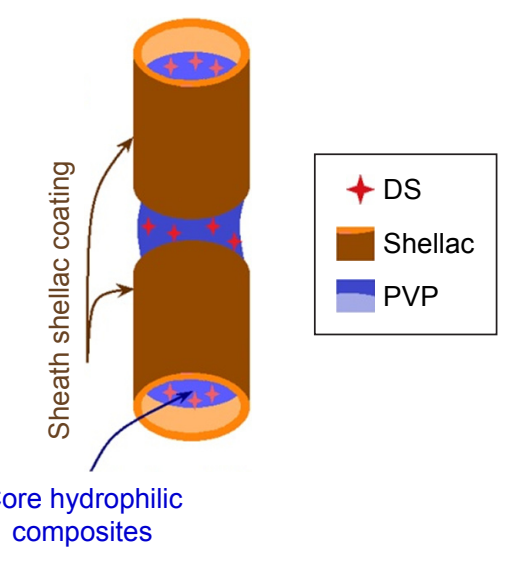

Figure I Schematic of the modified coaxial system (A) and the designed SCs with shellac nanocoating (B). Abbreviations: DS, diclofenac sodium; PVP, polyvinylpyrrolidone; SCs, structural nanocomposites. 

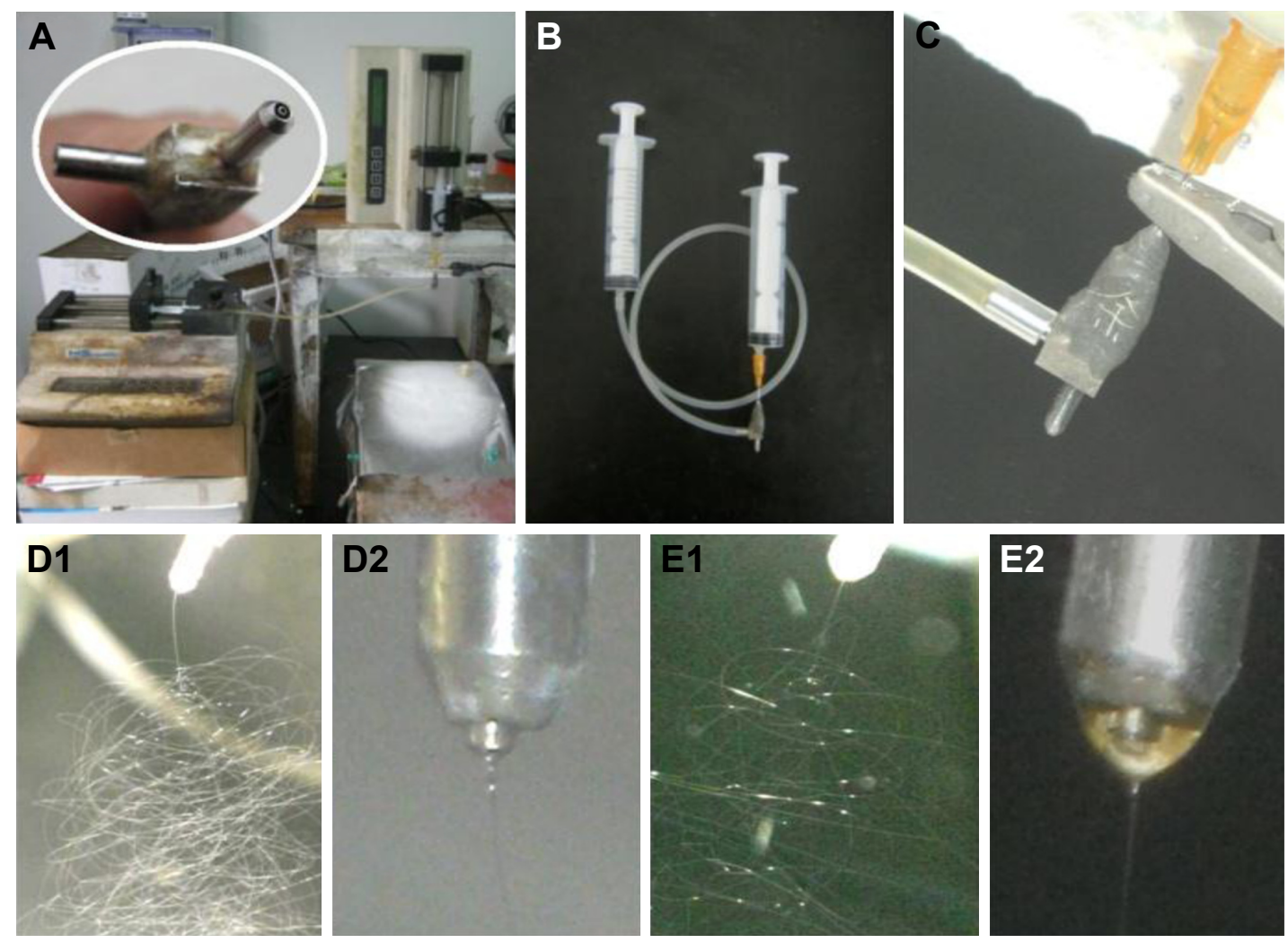

Figure 2 Implementations of the electrospinning processes.

Notes: (A) The whole electrospinning system. Inset shows the homemade coaxial spinneret; (B) the connections of spinneret with the syringes; (C) linkage of spinneret with the power supply; (DI and D2) typical images of the single-fluid blending electrospinning process and Taylor cone for preparing HC, respectively; and (EI and E2) typical images of the modified coaxial electrospinning process for fabricating SC.

Abbreviations: $\mathrm{HC}$, hydrophilic nanocomposites; SC, structural nanocomposite.
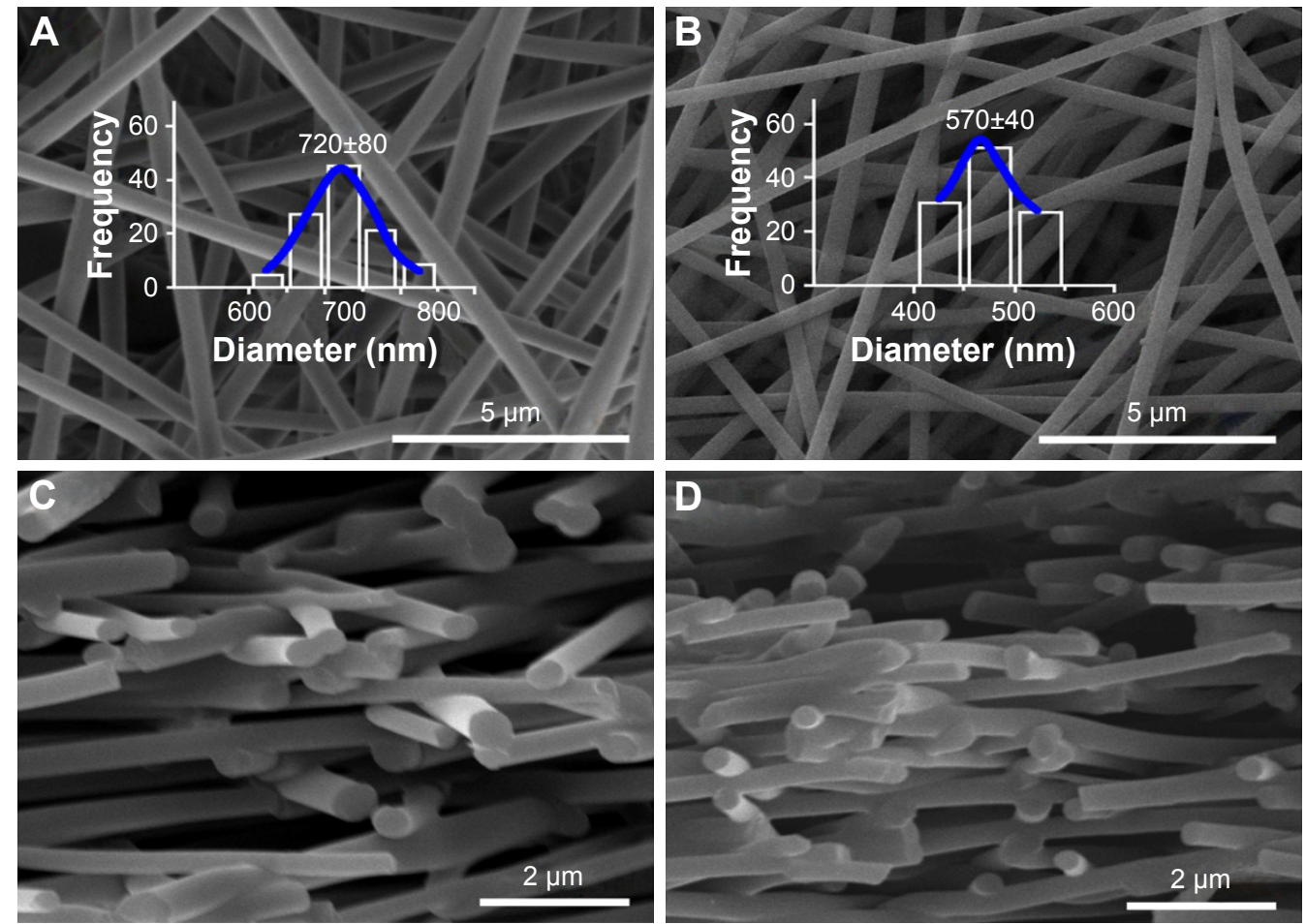

Figure 3 Scanning electron microscopy images of the surface morphologies of FI (A) and F2 nanofibers (B) and the cross sections of FI (C) and F2 (D), respectively. 
diameter of $720 \pm 80 \mathrm{~nm}$, whereas the core-sheath SC F2 nanofibers had an average diameter of $570 \pm 40 \mathrm{~nm}$. Their cross sections are exhibited in Figure $3 \mathrm{C}$ and D, respectively, which suggested that no solid phase separations occurred during both single-fluid and modified coaxial processes. The surface and cross-section morphologies revealed that F2 had a higher quality than F1 in terms of diameter and size distribution. The F2 nanofibers presented a smaller diameter and a narrower size distribution than F1. The dilute shellac sheath solution not only had a lower concentration than the core fluid, but it also extended the time at which the core solutions were drawn under the electrical field. The sheath shellac solution should resist possible negative influences from the environment for generating nanofibers stably and robustly. These factors acted together to produce core-shell SC nanofibers of high quality.

The TEM images of F1 and F2 nanofibers are shown in Figure 4A and B, respectively. F1 nanofibers had a monolithic structure, suggesting that they were homogeneous nanocomposites. By contrast, F2 nanofibers had distinct core-shell structures. Their sheath layer had an estimated thickness of $12 \mathrm{~nm}$. However, the core parts of F2 nanofibers had uniform structures without any separated particles, suggesting that they were core-sheath SCs with a shellac nanocoating on the core homogeneous composites.

\section{Physical form and compatibility}

Raw DS particles are crystalline materials, as suggested by the sharp peaks in its XRD patterns (Figure 5). By contrast, both shellac and PVP are amorphous polymeric carriers. After treatment by the two electrospinning processes, the working fluids were similarly converted into solid nanofibers. Both HCs and SCs were amorphous with two halos at their XRD patterns. These results suggested that the raw DS crystallines were distributed into the PVP matrices in a molecular manner in monolithic $\mathrm{HC}$ and, similarly, in the core parts of SC. The nanocoating processes by modified coaxial electrospinning did not influence the conversions of DS from the original crystalline state to amorphous state. Although a dilute solution was applied during the coaxial processes, rapid electrospinning still completed the drying processes at a timescale of $10 \mathrm{~ms}$. The extremely rapid processes duplicated the concentric structure of the spinneret for realizing a sheath coating. They also propagated into the core parts of the solid nanofibers, thereby altering the physical arrangement of the components in the core fluids and creating homogeneous core nanocomposites.

Compatibility between the guest drug and host matrices is crucial for the stability of nanocomposites. ${ }^{42}$ Therefore, ATR-FTIR experiments were conducted to detect the possible secondary interactions between the components. The spectra of the raw materials and their electrospun composites are shown in Figure 6. The raw DS powders had characteristic peaks at 1,578, 1,507, and 1,453 $\mathrm{cm}^{-1}$; these peaks were attributed to carbonyl groups and benzene rings. However, these typical peaks and also those in the finger regions of DS disappeared from the spectra of F1 and F2 nanofibers. This phenomenon demonstrated that no DS
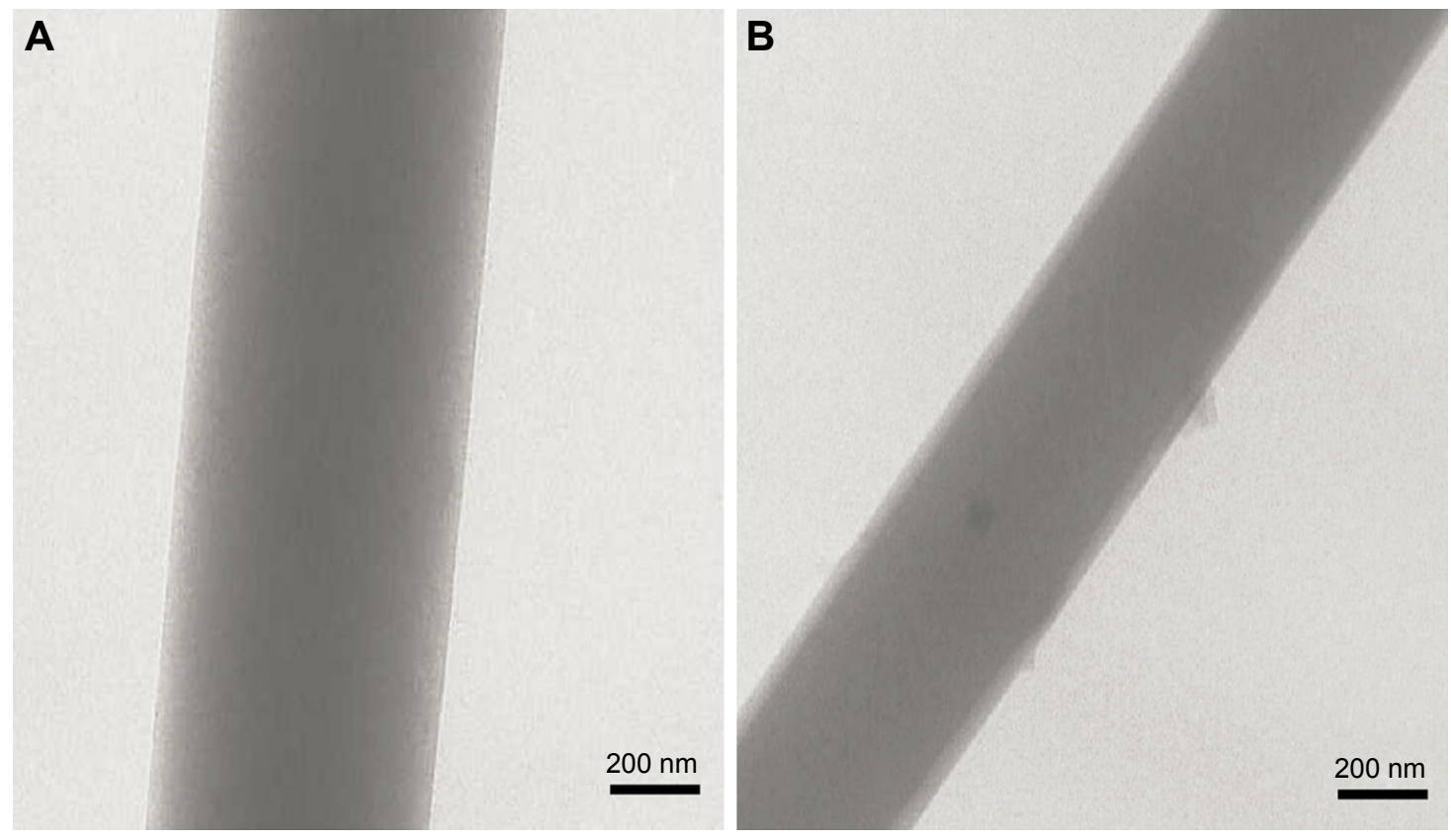

Figure 4 Transmission electron microscopy images of $\mathrm{FI}(\mathbf{A})$ and $\mathrm{F} 2$ nanofibers (B). 


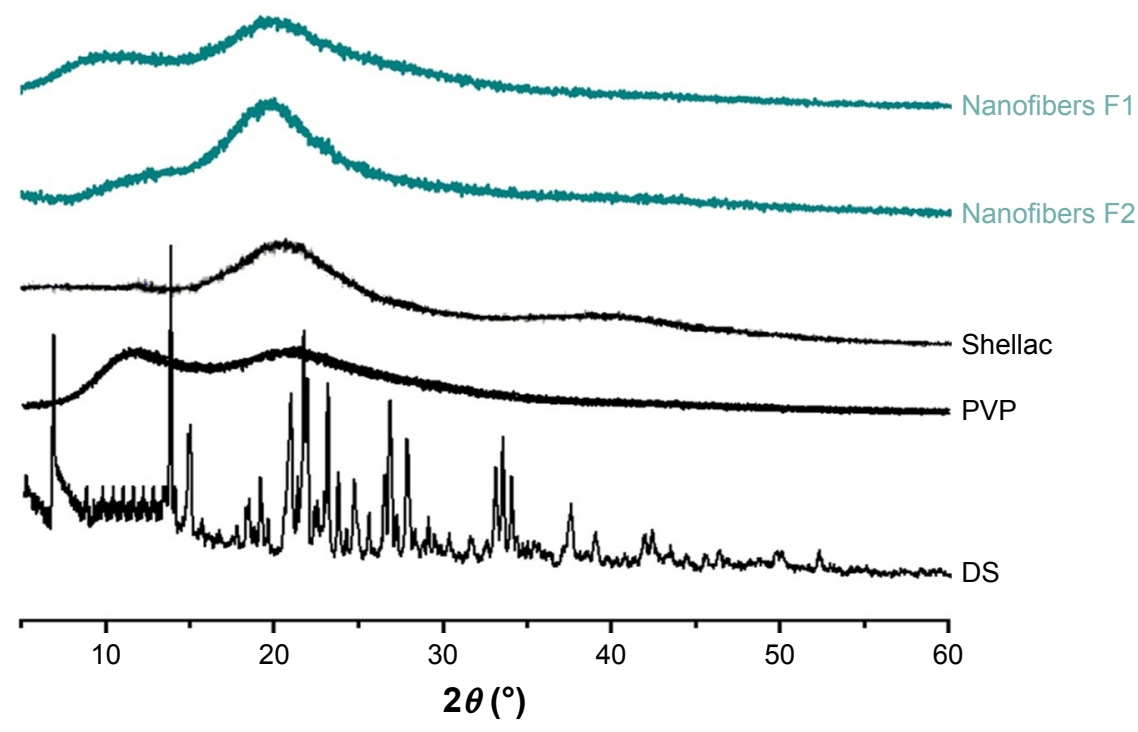

Figure 5 X-ray diffraction patterns.

Abbreviations: DS, diclofenac sodium; PVP, polyvinylpyrrolidone.

crystalline lattices existed in F1 and F2 nanofibers, revealing the fine compatibility between DS and PVP in the HC and the core parts of SC.

Additionally, the characteristic peaks of shellac at 1,702 and $1,087 \mathrm{~cm}^{-1}$ were very strong in the spectra of SC. These peaks suggest that $\mathrm{SC}$ is a "hybrid mixture" of shellac and DS-PVP composites, giving a hint of the core-sheath nanostructure. This situation may also be related to the detection depth of $100 \mathrm{~nm}$ in ATR-FTIR because the spectra of SC reflected the absorbance from the sheath shellac and partial core DS-PVP composites.

The chemical formula of raw materials is shown in Figure 6. The $\mathrm{C}=\mathrm{O}$ groups in $\mathrm{PVP}$ and $\mathrm{N}-\mathrm{H}$ groups in $\mathrm{DS}$ suggest that hydrogen bonds formed between DS and PVP molecules. Attractive electrostatic interactions should also occur between the negative and positive groups in the DS and PVP molecules. Hydrophobic interactions may occur between the long carbon chain of PVP and the benzene rings
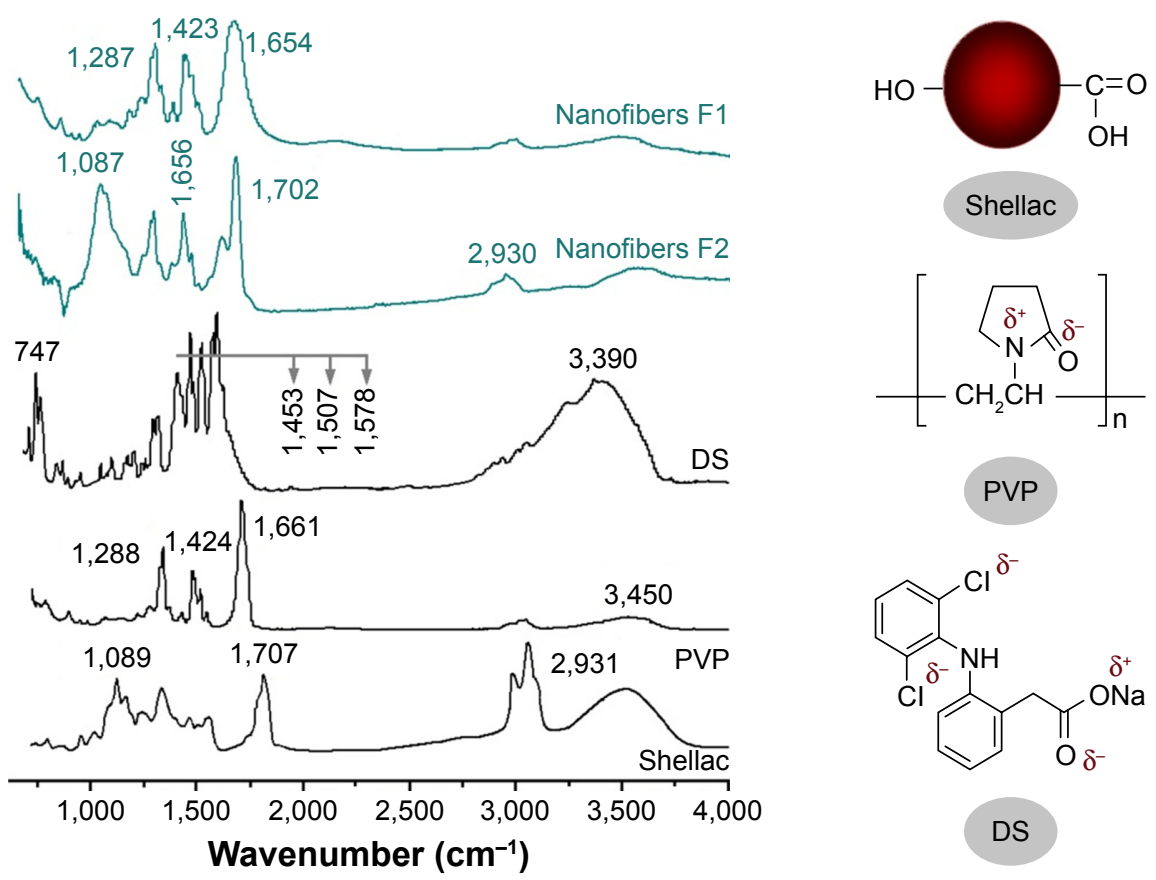

Figure 6 ATR-FTIR spectra and the molecular formula of components.

Abbreviations: ATR-FTIR, attenuated total reflectance-Fourier transform infrared; DS, diclofenac sodium; PVP, polyvinylpyrrolidone. 


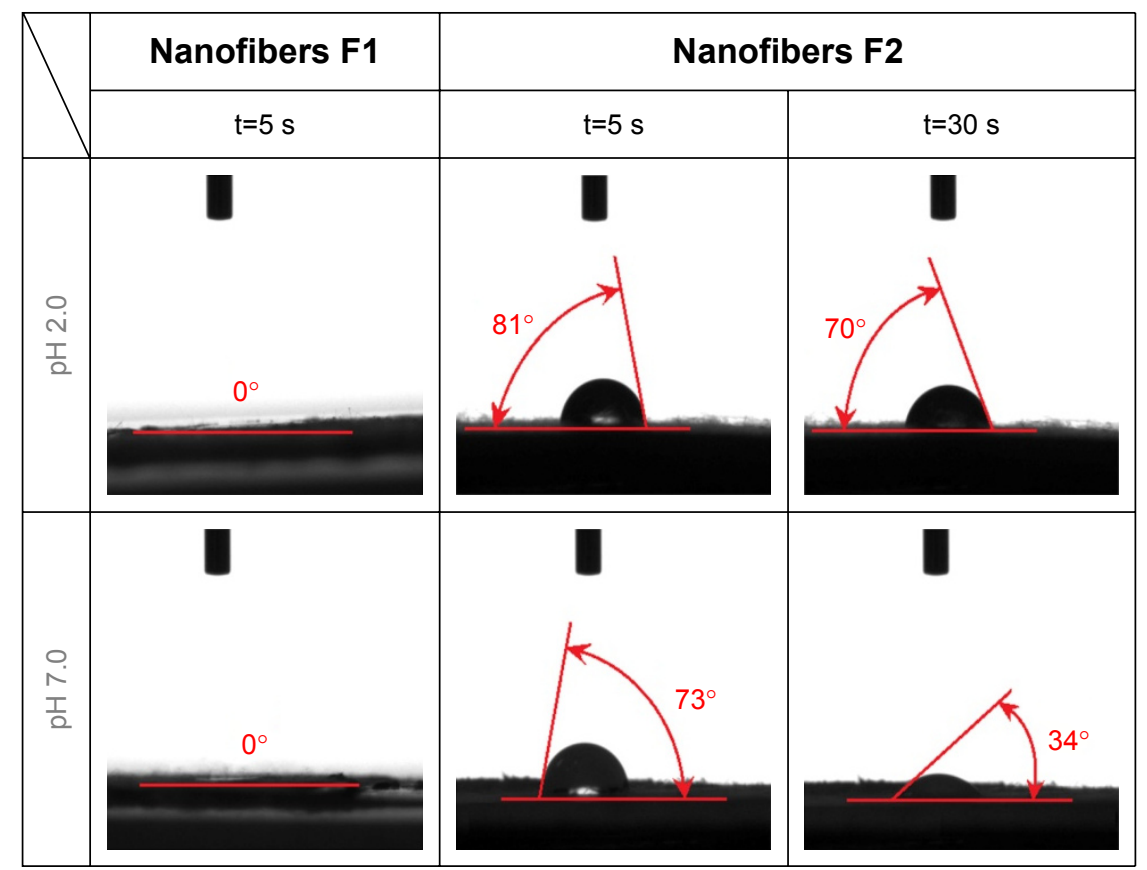

Figure 7 WCAs of HC (FI nanofibers) and SC (F2 nanofibers) using droplets with different $\mathrm{pH}$ values. Abbreviations: HC, hydrophilic nanocomposites; SC, structural nanocomposite; WCA, water contact angle.

of DS. Thus, secondary interactions, including hydrogen bonding, electrostatic interactions, and hydrophobic interactions, play a fundamental role in promoting the homogeneity of HC and core of SC.

\section{Hydrophilic properties}

WCAs of HCs and SCs were measured by placing $3 \mu \mathrm{L}$ droplets with different values on the nanofiber mats. As shown in Figure 7, the droplets disappeared after $5 \mathrm{~s}$ when they were placed on the top of $\mathrm{HCs}$ either at $\mathrm{pH} 2.0$ or 7.0. The PVP-based composites (or often called solid dispersions in pharmaceutics) have high hydrophilicity, which can promote the loaded poorly water-soluble drugs to be dissolved within several seconds. ${ }^{42,43}$ Thus, the drug may be released in a pulsatile manner in the mouth or stomach provided that these HCs are converted into solid dosage forms and administered orally.

The SCs presented significantly different performance when droplets with different values were applied. At $5 \mathrm{~s}$, the WCAs at pH 2.0 and 7.0 were $81^{\circ}$ and $73^{\circ}$, respectively. At $30 \mathrm{~s}$, the differences were evident, with values of $70^{\circ}$ and $34^{\circ}$ for acid and neutral conditions, respectively. These differences were closely related to the sheath shellac nanocoating, which could resist the acid droplets but could not reject the neutral liquid. The dissolution of shellac at $\mathrm{pH}$ 7.0 resulted in the rapid removal of droplets on SC.

\section{In vitro dissolution profiles}

As anticipated, the encapsulation efficiencies were around $100 \%$ for both the single-fluid and modified coaxial electrospinning products. Regardless of how many fluids were treated simultaneously via electrospinning, it is essentially a fluid-drying process with an extremely fast speed. During electrospinning, the evaporation of volatile ethanol did not result in the escape of drug molecules to the environment.

The in vitro drug-release profiles of the raw DS particles, $\mathrm{HCs}$, and SCs are exhibited in Figure 8. The raw drug particles freed the DS molecules very slowly because of the

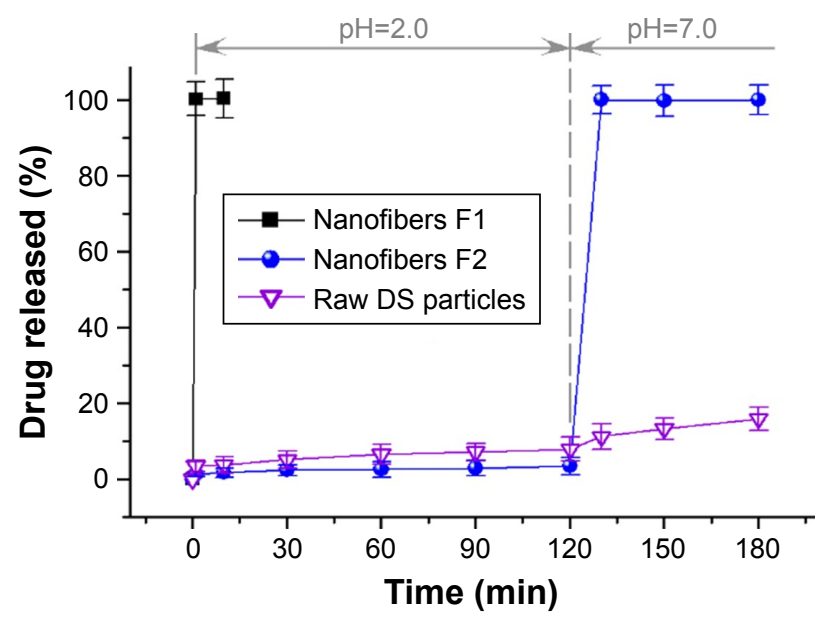

Figure 8 In vitro drug dissolution from the composites and raw DS particles. Abbreviation: DS, diclofenac sodium. 


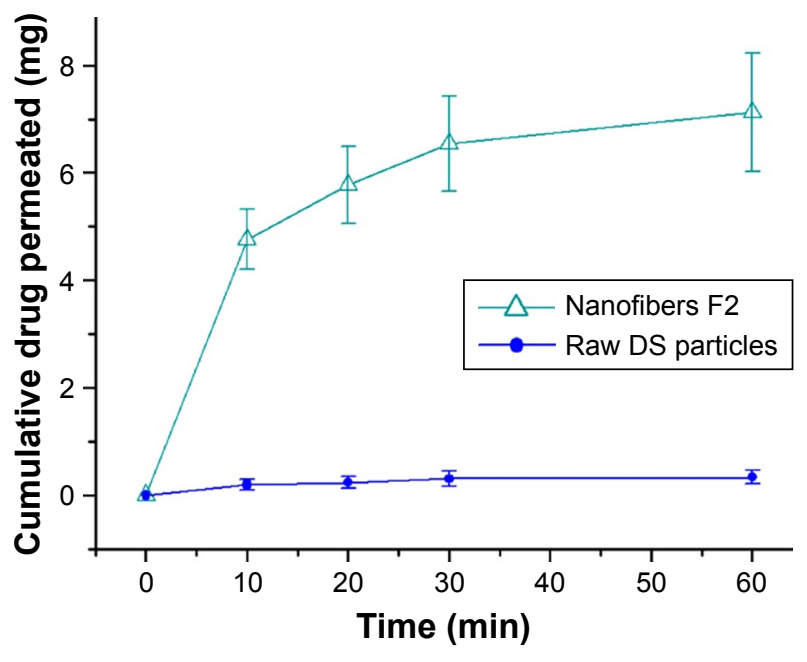

Figure 9 Ex vivo permeation profiles of SC and raw DS particles. Abbreviations: DS, diclofenac sodium; SC, structural nanocomposite.

high lattice energy. HCs disappeared to release the loaded DS all at once, providing a pulsatile release at $\mathrm{pH} 2.0$ dissolution media. By contrast, SCs released only $7.1 \% \pm 2.8 \%$ of its cargoes at the first $2 \mathrm{~h}$ but exhausted all the DS $10 \mathrm{~min}$ after it was transferred into neutral dissolution media. The insolubility of shellac enabled the core nanocomposites to resist low-pH environments in the simulated gastric fluids. In neutral-pH conditions of the simulated intestinal fluid, the SCs quickly disappeared and released the embedded DS, suggesting a typical colon-specific pulsatile drugrelease profile.

\section{Ex vivo permeation}

The permeation results are exhibited in Figure 9. DS, a biopharmaceutical class II drug, can easily penetrate fatty membranes but is difficult to dissolve. After $60 \mathrm{~min}$, only $0.34 \pm 0.13 \mathrm{mg}$ of the DS molecules was transmitted into the receptor cells. The slow dissolution of DS resulted in two negative influences on effective permeation. First, the amount of DS molecules in the donor cell was limited. Second, the concentration differences between the two cells were minimal. Thus, the passive transmembrane was weak. For the SC, $7.12 \pm 1.14 \mathrm{mg}$ of the DS molecules was transmitted into the receptor cells, indicating over 20 -fold permeation efficiency compared with the raw DS particles. This result reflects the situation of many commercial DS dosage forms, such as tablets from dry powder compression and capsules.

\section{Conclusion}

A core-shell SC was successfully prepared using modified coaxial electrospinning, by which a $\mathrm{pH}$-sensitive shellac was coated on amorphous HCs. Compared with the monolithic $\mathrm{HC}$ from single-fluid blending electrospinning, the SCs exhibited higher quality in terms of diameter and size distribution, as demonstrated by their SEM and TEM images. XRD patterns demonstrated that DS was converted into an amorphous status in the HCs and SCs because of the fine compatibility between PVP and DS. WCA experiments verified that the SCs could resist the erosion of acid conditions. In vitro dissolution tests verified that SCs could provide a typical colon-specific pulsatile release, rather than a pulsatile release of HCs in acid conditions. Ex vivo permeation tests demonstrated that the SCs provided an over 20 -fold drug permeation rate compared with the DS particles on the colon membrane. Nanostructures play important roles in designing new nanomedicines, and modified coaxial electrospinning is a useful tool for creating new nanostructures.

\section{Acknowledgment}

We are thankful for the financial support from the National Natural Science Foundation of China (No 51373101).

\section{Disclosure}

The authors report no conflicts of interest in this work.

\section{References}

1. Siepmann J, Peppas NA. Modeling of drug release from delivery systems based on hydroxypropyl methylcellulose (HPMC). Adv Drug Deliv Rev. 2012;64:163-174.

2. Fu QW, Zi YP, Xu W, et al. Electrospinning of calcium phosphate-poly (D, L-lactic acid) nanofibers for sustained release of water-soluble drug and fast mineralization. Int J Nanomedicine. 2016;11:5087-5097.

3. Reda RI, Wen MM, El-Kamel AH. Ketoprofen-loaded eudragit electrospun nanofibers for the treatment of oral mucositis. Int J Nanomedicine. 2017;12:2335-2351.

4. Hsu YH, Lin CT, Yu YH, Chou YC, Liu SJ, Chan EC. Dual delivery of active antibactericidal agents and bone morphogenetic protein at sustainable high concentrations using biodegradable sheath-corestructured drug-eluting nanofibers. Int J Nanomedicine. 2016;11: 3927-3937.

5. Brayden DJ. Controlled release technologies for drug delivery. Drug Discov Today. 2003;8(21):976-978.

6. Tseng YY, Yang TC, Wang Y C, et al. Targeted concurrent and sequential delivery of chemotherapeutic and antiangiogenic agents to the brain by using drug-loaded nanofibrous membranes. Int $J$ Nanomedicine. 2017;12:1265-1276.

7. Vemula SK, Kebamo S, Paulos B, et al. Formulation, development and evaluation of colon-specific ketorolac tromethamine compression coated tablets. Sci Technol Art Res J. 2015;4:150-154.

8. Vemula SK, Katkum R. Colon-specific double-compression coated pulsatile tablets of ketorolac tromethamine: formulation development and pharmacokinetics. J Drug Deliv Sci Technol. 2015;29:78-83.

9. Tung NT, Nguyen TH, Pham TT, et al. Pectin/HPMC dry powder coating formulations for colon specific targeting tablets of metronidazole. J Drug Deliv Sci Technol. 2016;33:19-27.

10. Vemula SK. A novel approach to flurbiprofen pulsatile colonic release: formulation and pharmacokinetics of double-compression-coated minitablets. AAPS Pharm Sci Tech. 2015;16(6):1465-1473. 
11. Vemula SK, Veerareddy PR, Devadasu VR. Pharmacokinetics of colon-specific $\mathrm{pH}$ and time-dependent flurbiprofen tablets. Eur J Drug Metabol Pharmacokinet. 2015;40(3):301-311.

12. Webster TJ. Nanomedicine: what's in a definition? Int J Nanomedicine. 2006;1(2):115-116.

13. Hassiba AJ, El Zowalaty ME, Nasrallah GK, et al. Review of recent research on biomedical applications of electrospun polymer nanofibers for improved wound healing. Nanomedicine (Lond). 2016;11(6): 715-737.

14. Hassiba AJ, El Zowalaty ME, Webster TJ, et al. Synthesis, characterization, and antimicrobial properties of novel double layer nanocomposite electrospun fibers for wound dressing applications. Int J Nanomedicine. 2017;12:2205-2213.

15. Ge L, Li Q, Jiang J, et al. Integration of nondegradable polystyrene and degradable gelatin in a core-sheath nanofibrous patch for pelvic reconstruction. Int J Nanomedicine. 2015;10:3193-3201.

16. Jalani G, Jung CW, Lee JS, Lim DW. Fabrication and characterization of anisotropic nanofiber scaffolds for advanced drug delivery systems. Int J Nanomedicine. 2014;9(Suppl 1):33-49.

17. Jung CW, Jalani G, Ko J, Choo J, Lim DW. Synthesis, characterization, and directional binding of anisotropic biohybrid microparticles for multiplexed biosensing. Macromol Rapid Commun. 2014;35(1):56-65.

18. Liu ZP, Cui L, Yu DG, Zhao ZX, Chen L. Electrosprayed core-shell solid dispersions of acyclovir fabricated using an epoxy-coated concentric spray head. Int J Nanomedicine. 2014;9:1967-1977.

19. Chen G, Xu Y, Yu DG, Zhang DF, Chatterton NP, White KN. Structuretunable Janus fibers fabricated using spinnerets with varying port angles. Chem Commun (Camb). 2015;51(22):4623-4626.

20. Bacakova M, Pajorova J, Stranska D, et al. Protein nanocoatings on synthetic polymeric nanofibrous membranes designed as carriers for skin cells. Int J Nanomedicine. 2017;12:1143-1160.

21. Zhou T, Sui B, Mo X, Sun J. Multifunctional and biomimetic fish collagen/bioactive glass nanofibers: fabrication, antibacterial activity and inducing skin regeneration in vitro and in vivo. Int J Nanomedicine. 2017;12:3495-3507.

22. Xu Y, Li JJ, Yu DG, Williams GR, Yang JH, Wang X. Influence of the drug distribution in electrospun gliadin fibers on drug-release behavior. Eur J Pharm Sci. 2017;106:422-430.

23. Ghosh Chaudhuri R, Paria S. Core/shell nanoparticles: classes, properties, synthesis mechanisms, characterization, and applications. Chem Rev. 2012;112(4):2373-2433.

24. Yu DG, White K, Chatterton N, Li Y, Li L, Wang X. Structural lipid nanoparticles self-assembled from electrospun core-shell polymeric nanocomposites. RSC Adv. 2015;5(13):9462-9466.

25. Lee $\mathrm{CH}$, Chao YK, Chang SH, et al. Nanofibrous rhPDGF-eluting PLGA-collagen hybrid scaffolds enhance healing of diabetic wounds. RSC Adv. 2016;6(8):6276-6284.

26. Bacakova L, Bacakova M, Pajorova J, et al. Nanofibrous scaffolds as promising cell carriers for tissue engineering. In: Nanofiber ResearchReaching New Heights. InTech Open; 2016; Chapter 3:29-53.
27. Li JJ, Yang C, Li HP, et al. Oral controlled release in accordance with drug adsorption biological rhythm provided by an electrospun structural amorphous solid dispersion. J Control Release. 2017;259:e61-e62.

28. Yu DG, Li JJ, Zhang M, Williams GR. High-quality Janus nanofibers prepared using three-fluid electrospinning. Chem Commun (Camb). 2017;53(33):4542-4545.

29. Yu DG, Li XY, Wang X, Yang JH, Bligh SW, Williams GR. Nanofibers fabricated using triaxial electrospinning as zero order drug delivery systems. ACS Appl Mater Interfaces. 2015;7(33):18891-18897.

30. Cetin M, Atila A, Kadioglu Y. Formulation and in vitro characterization of Eudragit L100 and Eudragit L100-PLGA nanoparticles containing diclofenac sodium. AAPS Pharm Sci Technol. 2010;11(3): 1250-1256.

31. Xu K, Liu Y, Bu S, et al. Egg albumen as a fast and strong medical adhesive glue. Adv Healthc Mater. 2017;6(19). Epub 2017 Jul 17.

32. Li XY, Shi CJ, Yu DG, Liao YZ, Wang X. Electrospun quercetin-loaded zein nanoribbons. Biomed Mater Eng. 2014;24(6):2015-2023.

33. Locertales IG, Barrero A, Guerrero I, Cortijo R, Marquez M, GañánCalvo AM. Micro/nano encapsulation via electrified coaxial liquid jets. Science. 2002;295(5560):1695-1698.

34. Dzenis Y. Spinning continuous fibers for nanotechnology. Science. 2004;304(5679):1917-1919.

35. Moghe K, Gupta BS. Co-axial electrospinning for nanofiber structures: preparation and applications. Polym Rev. 2008;48(2):353-377.

36. Wang Q, Yu DG, Zhang LL, Liu XK, Deng YC, Zhao M. Electrospun hypromellose-based hydrophilic composites for rapid dissolution of poorly water-soluble drug. Carbohydr Polym. 2017;174:617-625.

37. Xie J, Mao H, Yu DG, et al. Highly stable coated polyvinylpyrrolidone nanofibers prepared using modified coaxial electrospinning. Fiber Polym. 2014;15(1):78-83.

38. Yang GZ, Li JJ, Yu DG, He MF, Yang JH, Williams GR. Nanosized sustained-release drug depots fabricated using modified tri-axial electrospinning. Acta Biomater. 2017;53:233-241.

39. Wang Q, Li HP, Yang C, et al. Beads-on-a-string amorphous solid dispersion fabricated using a modified coaxial electrospinning. $J$ Control Release. 2017;259:e111-e112.

40. Yu DG, Li HP, Yang C, et al. Double-pulsatile release core-shell fibers fabricated using modified tri-axial electrospinning. J Control Release. 2017;259:e24-e25.

41. Wang X, Yu DG, Li XY, Bligh SW, Williams GR. Electrospun medicated shellac nanofibers for colon-targeted drug delivery. Int J Pharm. 2015;490(1-2):384-390.

42. Yan J, White K, Yu DG, Zhao XY. Sustained release multiple-component cellulose acetate nanofibers fabricated using a modified coaxial electrospinning process. J Mater Sci. 2014;49(2):538-547.

43. Yu DG, Shen XX, Brandford-White C, White K, Zhu LM, Bligh SW. Oral fast-dissolving drug delivery membranes prepared from electrospun polyvinylpyrrolidone ultrafine fibers. Nanotechnology. 2009; 20(5):055104
International Journal of Nanomedicine

\section{Publish your work in this journal}

The International Journal of Nanomedicine is an international, peerreviewed journal focusing on the application of nanotechnology in diagnostics, therapeutics, and drug delivery systems throughout the biomedical field. This journal is indexed on PubMed Central, MedLine, CAS, SciSearch ${ }^{\circledR}$, Current Contents ${ }^{\circledR} /$ Clinical Medicine,

\section{Dovepress}

Journal Citation Reports/Science Edition, EMBase, Scopus and the Elsevier Bibliographic databases. The manuscript management system is completely online and includes a very quick and fair peer-review system, which is all easy to use. Visit http://www.dovepress.com/ testimonials.php to read real quotes from published authors. 